\title{
JEJUM PRÉ-OPERATÓRIO NO CLIENTE SUBMETIDO A CIRURGIA ELETIVA: REVISÃO INTEGRATIVA DA LITERATURA
}

Pre-operative fast in patients subjected to elective surgery: integrative literature review

Ayuno preoperatorio en el cliente presentado a la cirugía electiva: revisión integrativa de la literatura

Susana Castro, ${ }^{*}$ João Ventura-Silva, ${ }^{* *}$ Olga Ribeiro ${ }^{* * *}$

\section{RESUMO}

Enquadramento: a implementação do jejum pré-operatório tem sido uma preocupação mundial dos vários profissionais de saúde, havendo uma atuação distinta, no que concerne às orientações em vigor em cada uma das instituições. Objetivos: conhecer a evidência científica sobre as orientações atuais acerca da aplicação do jejum pré-operatório em clientes submetidos a cirurgia eletiva, publicada nos últimos cinco anos. Metodologia: estudo de revisão integrativa da literatura

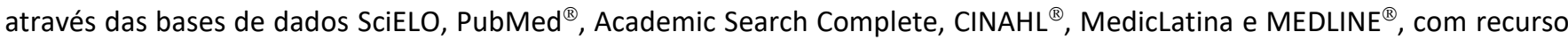
aos descritores "fasting", "preoperative care", "patients" e "nursing". Resultados: foram analisados dez artigos e os dados organizados em três macrocategorias: orientações sobre o jejum pré-operatório; ganhos inerentes à implementação das orientações atuais; e fatores dificultadores da implementação das orientações atuais sobre o jejum pré-operatório. Conclusão: as várias organizações que se debruçam sobre esta temática já evidenciaram guidelines norteadoras. Contudo nas instituições de saúde nem sempre se verifica a implementação destas orientações. O enfermeiro deverá consciencializar o cliente deste procedimento pré-operatório, assim como ser um agente de mudança no cumprimento deste procedimento.

Palavras-Chave: jejum; cuidados pré-operatórios; pacientes; enfermagem

*MsC, Enfermeira Bloco Operatório CentralInstituto Português de Oncologia do Porto https://orcid.org/0000-0001-8864-8634

autor de correspondência:

enf.susanacastro@gmail.com

${ }^{* *} \mathrm{MsC}$, Enfermeiro Especialista em Enfermagem de Reabilitação. Centro Hospitalar de São João EPE: Porto

https://orcid.org/0000-0002-8794-528X

${ }^{* * *}$ PhD, Professora adjunta, Escola Superior de Enfermagem do Porto

https://orcid.org/0000-0001-9982-9537
Como referenciar:

Castro, S., Ventura-Silva, J., \& Ribeiro, O. (2020) Jejum pré-operatório no cliente submetido a cirurgia eletiva: revisão integrativa da literatura. Revista de Investigação \& Inovação em Saúde, 3(2), 75-85. doi.org/10.37914/riis.v3i2.75
Recebido para publicação em: 29/03/2020 Aceite para publicação em: 24/07/2020

\section{ABSTRACT}

Background: the implementation of preoperative fasting has been a worldwide concern of the various health professionals, with a different role, with regard to the guidelines in force in each of the institutions. Objectives: to know the scientific evidence on the current guidelines on the application of preoperative fasting in clients undergoing elective surgery, published in the last five years. Methodology: an integrative literature review study using the SciELO, PubMed ${ }^{\circledR}$, Academic Search Complete, CINAHL $^{\circledR}$, MedicLatina and MEDLINE ${ }^{\circledR}$ databases, using the keywords "fasting", "preoperative care", "patients" and "nursing". Results: ten articles were analyzed and the data were organized into three macro categories: guidance on preoperative fasting; gains inherent in the implementation of the current guidelines; and factors that hinder the implementation of current guidelines on preoperative fasting. Conclusion: the various organizations that deal with this theme have already shown guiding guidelines. However, health institutions do not always implement these guidelines. The nurse must make the client aware of this preoperative procedure, as well as be an agent of change in complying with this procedure.

Keywords: fasting; preoperative care; patients; nursing

\section{RESUMEN}

Marco contextual: la implementación del ayuno preoperatorio ha sido una preocupación mundial para los diversos profesionales de la salud, con un papel diferente con respecto a las pautas vigentes en cada una de las instituciones. Objetivos: conocer la evidencia científica sobre las pautas actuales sobre la aplicación del ayuno preoperatorio en clientes sometidos a cirugía electiva, publicada en los últimos cinco años. Metodología: un estudio integrador de revisión de literatura que utiliza las bases de datos SciELO, PubMed ${ }^{\circledR}$, Academic Search Complete, CINAHL ${ }^{\circledR}$, MedicLatina y MEDLINE ${ }^{\circledR}$, utilizando las palabras clave "ayuno", "atención preoperatoria", "pacientes" y "enfermería". Resultados: se analizaron diez artículos y los datos se organizaron en tres categorías macro: orientación sobre el ayuno preoperatorio; ganancias inherentes a la implementación de las directrices actuales; y factores que dificultan la implementación de las pautas actuales sobre el ayuno preoperatorio. Conclusión: las diversas organizaciones que se ocupan de este tema ya han mostrado pautas orientativas. Sin embargo, las instituciones de salud no siempre implementan estas pautas. La enfermera debe informar al cliente sobre este procedimiento preoperatorio, así como ser un agente de cambio para cumplir con este procedimiento.

Palabras Clave: ayuno; cuidados preoperatorios; pacientes; enfermería 
Jejum pré-operatório no cliente submetido a cirurgia eletiva: revisão integrativa da literatura

\section{INTRODUÇÃO}

No contexto nacional e internacional, a realização de cirurgias eletivas tem tido um aumento exponencial e com uma complexidade cada vez maior, envolvendo procedimentos de grande abordagem, multiorgânicos, de longa duração e em clientes com morbilidades múltiplas. A pessoa intervencionada durante a sua cirurgia desencadeia uma série de respostas orgânicas, devido ao traumatismo provocado, com o intuito de reparar os tecidos lesados e repor a homeostase corporal, e que se agrava com o jejum pré-operatório prolongado (Francisco, Batista, \& Pena, 2015). A conjugação destes dois elementos, traumatismo cirúrgico e o jejum prolongado, gera uma diminuição dos níveis de insulina, um aumento do cortisol e do glucagon, da resposta inflamatória e da produção de catecolaminas que, por sua vez, produzirão um aumento na resistência à insulina que começa na fase intraoperatória e se estende no pós-operatório. Ao mesmo tempo surge a ativação do processo de neoglicogénese, aumentando a glicemia sanguínea o que intensifica o stresse metabólico cirúrgico (Yildiz, Gunal, Yilmaz, \& Yucel, 2013).

Sendo o jejum pré-operatório a abstinência de alimentos e líquidos num período de tempo que antecede a indução anestésica e a cirurgia, Powers (2017) reporta ainda que atualmente são dadas indicações para o cliente adotar o modelo "nada pela boca" (NPO), que consiste numa interrupção da ingestão destes alimentos desde as zero horas anteriores à cirurgia programada. É ainda aludido que com esta prática a prevenção de possíveis complicações na fase intraoperatória como o vómito e a aspiração de conteúdo gástrico durante a indução anestésica e o procedimento cirúrgico (Çakar, Yilmaz,
Çakar, \& Baydur, 2017). No entanto, já em 1999, com reformulações em 2011, a American Society of Anaesthesiologists (ASA) desaconselhava um jejum pré-operatório tão prolongado, assegurando que este comportava mais prejuízos do que ganhos para o cliente, uma vez que aumentava o stresse cirúrgico e respostas fisiológicas indesejáveis, como a desidratação, a ansiedade, a fome, a sede, a xerostomia, a fadiga, a inquietação, as cefaleias, o desconforto pré e pós-operatório, implicando um défice na reserva de fluídos para o período pósoperatório (Macqbali, 2016). Contudo, em 2014, Aguilar-Nascimento, Dias, Dock-Nascimento, Correia, Campos, Portari-Filho \& Oliveira ainda referiam que o jejum prolongado era uma prática continuada nos cuidados pré-operatórios, apesar de já existirem algumas orientações contrárias a esse procedimento. Outros autores acrescentavam que a supressão do tempo de jejum prolongado poderia influenciar positivamente a redução do tempo de internamento pós-operatório e as complicações e intercorrências derivadas da intervenção cirúrgica (Pinto, Grigoletti \& Marcadenti, 2015). Neste contexto, no ano de 2019, o protocolo Enhanced Recovery After Surgery (ERAS) definiu intervenções multidisciplinares para os vários momentos operatórios, contemplando a prevenção do jejum prolongado, tendo em vista uma diminuição do stresse cirúrgico e uma aceleração da recuperação pósoperatória num contexto de segurança para o cliente. Atendendo ao exercício profissional do enfermeiro, que também se centra neste domínio, é-lhe exigido uma conceção e implementação de cuidados, baseados na melhor evidência científica, e adequados às caraterísticas clínicas do cliente a intervencionar, com o intuito de minimizar o impacto da cirurgia e 
Jejum pré-operatório no cliente submetido a cirurgia eletiva: revisão integrativa da literatura

promover a obtenção de resultados anestésicocirúrgicos positivos. Todavia, nos vários contextos da prática clínica, nem sempre a implementação do jejum pré-operatório é orientada nos pressupostos apresentados. Imbuídos nesta convicção, este estudo tem como objetivo conhecer a evidência científica sobre as orientações atuais acerca da aplicação do jejum pré-operatório em clientes submetidos a cirurgia eletiva, publicada nos últimos cinco anos.

\section{PROCEDIMENTOS METODOLÓGICOS DE REVISÃO}

Trata-se de um estudo de revisão integrativa da literatura, que potencia a síntese de todos os achados disponíveis sobre a temática, permitindo nortear o exercício profissional do enfermeiro e sustentá-lo em conhecimento científico (Souza, Silva, \& Carvalho, 2010).

A revisão integrativa da literatura possibilita a combinação da investigação primária e secundária, após a avaliação da sua qualidade metodológica (Sousa, Firmino, Marques-Vieira, Severino, \& Pestana, 2018) estando direcionada para a prática baseada na evidência, permitindo a inclusão das evidências nos contextos práticos com segurança (Vilelas, 2017). Na sua elaboração teve-se por referência as seis etapas preconizadas por Botelho, Cunha \& Macedo (2011) correspondentes à determinação do tema de estudo, formulação da questão de investigação, determinação dos critérios de inclusão e exclusão, identificação dos estudos selecionados, categorização dos mesmos, análise e interpretação dos achados a incluir na revisão e apresentação da revisão/síntese do conhecimento produzido e publicado.

Delineou-se como questão norteadora do estudo: "Quais as orientações atuais sobre o jejum pré- operatório em clientes submetidos a cirurgia eletiva, publicadas nos últimos cinco anos?", com recurso à estratégia PICO. Neste estudo os participantes (P) são os clientes submetidos a cirurgia eletiva (pacientes), a intervenção (I) que diz respeito ao jejum préoperatório; a comparação (C), não é aplicável no estudo, e os resultados (outcomes) relativos às orientações (cuidados pré-operatórios). A colheita de dados ocorreu durante o mês de fevereiro de 2020 nas bases de dados Scientific Electronic Library Online (SciELO), PubMed ${ }^{\circledR}$ e plataforma EBSCOhost WEB onde se englobaram a Academic Search Complete, $\mathrm{CINAHL}^{\circledR}$ Complete, CINAHL ${ }^{\circledR}$ Plus with Full Text, MedicLatina, MEDLINE ${ }^{\circledR}$ e MEDLINE ${ }^{\circledR}$ with Full Text, com recurso aos descritores "fasting", "preoperative care", "patients" e "nursing" também em Língua portuguesa e espanhola.

A frase booleana do estudo foi "fasting" AND "preoperative care" AND "patients" AND "nursing". Os critérios de inclusão definidos na pesquisa foram publicações nos idiomas de português, inglês e espanhol, no período de janeiro de 2015 a janeiro de 2020 e disponíveis em texto integral. Como critérios de exclusão, delinearam-se os estudos referentes à população pediátrica.

Da pesquisa elaborada resultaram 38 artigos. Numa primeira fase, procedeu-se a uma leitura críticoreflexiva dos títulos por dois investigadores, seguindose a leitura dos resumos e estudos encontrados, por três investigadores de forma independente.

Atendendo aos critérios de inclusão e exclusão e à avaliação da qualidade metodológica estabeleceu-se uma amostra de 10 artigos. A figura 1 apresenta o procedimento de seleção dos artigos a analisar, segundo a metodologia PRISMA (Galvão, Pansani, \& Harrad, 2015). 

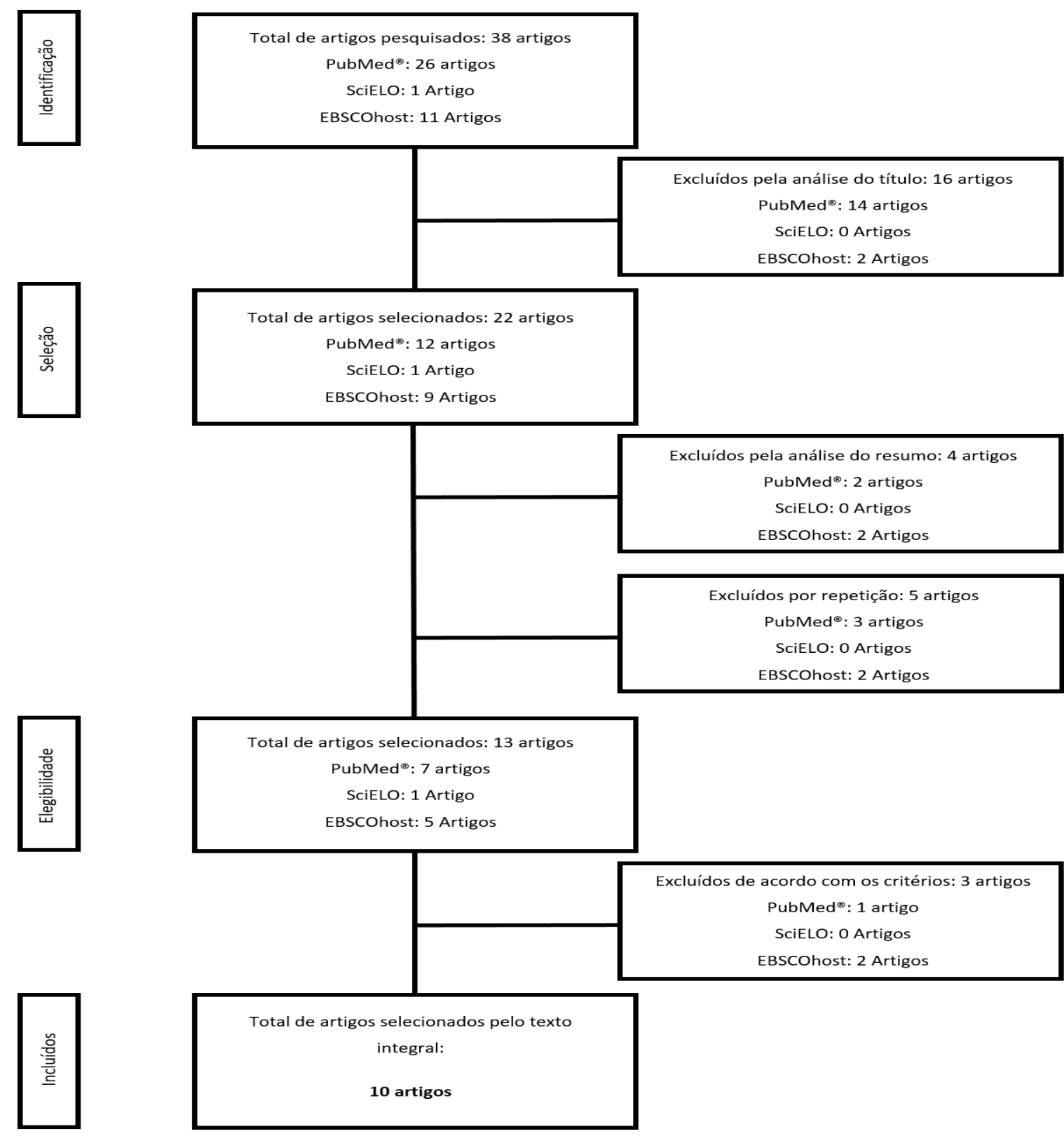

Figura 1

Processo redutivo da revisão integrativa

Com o intuito de facilitar a análise de cada artigo, optou-se pela identificação, tradução e transcrição de todos os aspetos relacionados com o jejum préoperatório. De forma a organizar a informação extraída dos artigos, elaborou-se uma tabela que permitiu a identificação e revisão das categorias relacionadas com a temática em estudo. A tabela 1 apresenta os estudos selecionados para análise. 
Tabela 1

Síntese dos artigos selecionados para análise

\begin{tabular}{|c|c|c|c|}
\hline Autores/Ano & Título & Metodologia & Resultados \\
\hline $\begin{array}{l}\text { Noba, L., } \quad \& \\
\text { Wakefield, A./ } \\
2019\end{array}$ & $\begin{array}{l}\text { Are carbohydrate } \\
\text { drinks more efective } \\
\text { than preoperative } \\
\text { fasting: A systematic } \\
\text { review of randomised } \\
\text { controlled trials }\end{array}$ & $\begin{array}{l}\text { Revisão sistemática da } \\
\text { literatura de } 22 \text { estudos } \\
\text { randomizados } \\
\text { controlados, envolvendo } \\
2065 \text { clientes }\end{array}$ & $\begin{array}{l}\text { A implementação de bebidas com } \\
\text { carboidratos no pré-operatório pode gerar } \\
\text { redução do tempo de internamento } \\
\text { hospitalar, reduz significativamente a } \\
\text { resistência à insulina e o desconforto pós- } \\
\text { operatório e não aumenta o risco de } \\
\text { broncoaspiração. É segura a ingestão destas } \\
\text { bebidas até } 2 \text { horas antes da cirurgia. }\end{array}$ \\
\hline $\begin{array}{l}\text { Munsterman, } \\
\text { C., \& Strauss, P. } \\
\text { / } 2018\end{array}$ & $\begin{array}{l}\text { Early rehydratation in } \\
\text { surgical patients with } \\
\text { prolonged fasting } \\
\text { decreases } \\
\text { postoperative nausea } \\
\text { and vomiting }\end{array}$ & $\begin{array}{l}\text { Estudo observacional, } \\
\text { descritivo, caso-controle, } \\
\text { envolvendo } 381 \text { adultos } \\
\text { submetidos a jejum pré- } \\
\text { operatório. }\end{array}$ & $\begin{array}{l}\text { Os clientes sujeitos a jejum prolongado antes } \\
\text { da cirurgia e a reforço da hidratação, } \\
\text { apresentaram uma taxa de } 4.25 \% \text { de náuseas } \\
\text { e vómitos no pós-operatório em comparação } \\
\text { com a taxa de } 14.6 \% \text { para aqueles que não } \\
\text { usufruíram do reforço hídrico. }\end{array}$ \\
\hline $\begin{array}{lrr}\text { Çakar, } & \text { E., } \\
\text { Yilmaz, } & \text { E., } \\
\text { Çakar, } & \text { E., } & \text { \& } \\
\text { Baydur, } & & \text { H. } \\
\text { /2017 } & & \end{array}$ & $\begin{array}{l}\text { The effect of } \\
\text { preoperative oral } \\
\text { carbohydrate solution } \\
\text { intake on patient } \\
\text { Comfort: A randomised } \\
\text { controlled study }\end{array}$ & $\begin{array}{ll}\text { Estudo randomizado } \\
\text { controlado incluindo } 90 \\
\text { clientes sujeitos a } \\
\text { tiroidectomia. }\end{array}$ & $\begin{array}{l}\text { Pessoas não sujeitas à ingestão de bebidas } \\
\text { ricas em carboidratos apresentaram mais } \\
\text { sede, fome, xerostomia, cefaleias, náuseas, } \\
\text { vómitos, desconforto em relação às que } \\
\text { ingeriram bebidas com caboidratos. } \\
\text { Há uma diferença significativa na pressão } \\
\text { arterial diastólica e frequência cardíaca na } \\
\text { fase intraoperatória. Clientes que ingeriram } \\
\text { carboidratos têm maior pressão arterial } \\
\text { diastólica e menor frequência cardíaca. }\end{array}$ \\
\hline $\begin{array}{l}\text { Njoroge, G., } \\
\text { Kivuti-Bitok, L., } \\
\text { \& Kimani, S. / } \\
2017\end{array}$ & $\begin{array}{l}\text { Preoperative } \text { fasting } \\
\text { among adult patients } \\
\text { for elective surgery in a } \\
\text { Kenyan referral } \\
\text { hospital }\end{array}$ & $\begin{array}{l}\text { Estudo observacional, } \\
\text { transversal a envolver } 65 \\
\text { pessoas sujeitas a cirurgia }\end{array}$ & $\begin{array}{l}93.8 \% \text { das pessoas têm um reduzido } \\
\text { conhecimento sobre os cuidados para o } \\
\text { cumprimento do tempo de jejum pré- } \\
\text { operatório adequado, permanecendo } 73.8 \% \\
\text { desses deles mais de } 15 \text { horas sem a ingestão } \\
\text { de sólidos e líquidos. As orientações sobre o } \\
\text { jejum pré-operatório são realizadas em } 80 \% \\
\text { dos casos por enfermeiros. }\end{array}$ \\
\hline $\begin{array}{l}\text { Powers, J. / } \\
2017\end{array}$ & $\begin{array}{l}\text { Guidelines for } \\
\text { preoperative fasting for } \\
\text { hospitalized patients }\end{array}$ & Artigo de reflexão & $\begin{array}{l}\text { A suspensão da nutrição entérica antes de } \\
\text { uma cirurgia deve ser minimizada para evitar } \\
\text { o compromisso nutricional na pessoa. É } \\
\text { seguro suspendê-la o mais próximo possível } \\
\text { da cirurgia, mediante condição clínica da } \\
\text { pessoa. }\end{array}$ \\
\hline $\begin{array}{l}\text { Sánchez, A., \& } \\
\text { Papapietro, K. / } \\
2017\end{array}$ & $\begin{array}{l}\text { Nutrición } \\
\text { perioperatoria en } \\
\text { protocolos quirúrgicos } \\
\text { para una mejor } \\
\text { recuperación } \\
\text { postoperatoria } \\
\text { (Protocolo ERAS) }\end{array}$ & $\begin{array}{l}\text { Revisão narrativa } \mathrm{da} \\
\text { literatura }\end{array}$ & $\begin{array}{l}\text { A nutrição perioperatória antes da cirurgia } \\
\text { contempla a abolição de um período } \\
\text { prolongado de jejum e a ingestão de soluções } \\
\text { com carboidratos até duas horas antes do } \\
\text { procedimento. Isto reduz as complicações e } \\
\text { internamento pós-operatório. }\end{array}$ \\
\hline $\begin{array}{l}\text { Abdullah } \quad \text { Al } \\
\text { Maqbali, M. / } \\
2016\end{array}$ & $\begin{array}{l}\text { Preoperative fasting for } \\
\text { elective surgery in a } \\
\text { regional hospital in } \\
\text { Oman }\end{array}$ & $\begin{array}{l}\text { Estudo de coorte, } \\
\text { longitudinal, prospetivo e } \\
\text { observacional, incluindo } \\
169 \text { clientes cirúrgicos. }\end{array}$ & $\begin{array}{l}\text { As pessoas estudadas revelam ter tido um } \\
\text { período de jejum pré-operatório } \\
\text { compreendido entre as } 7 \text { horas e } 19 \text { horas } \\
\text { para alimentos sólidos e de } 4 \text { horas a } 19 \text { horas } \\
\text { para líquidos, ultrapassando as } \\
\text { recomendações. Estes acontecimentos geram } \\
\text { mais desconforto e potenciam a morbilidade. }\end{array}$ \\
\hline
\end{tabular}




\begin{tabular}{|c|c|c|c|}
\hline $\begin{array}{l}\text { Vidot, H., } \\
\text { Teevan, K., } \\
\text { Carey, } \\
\text { Strasser, S., \& } \\
\text { Shackel, N. / } \\
2016\end{array}$ & $\begin{array}{l}\text { A prospective audit of } \\
\text { preprocedural fasting } \\
\text { practices on a } \\
\text { transplant ward: when } \\
\text { fasting becomes } \\
\text { starving }\end{array}$ & $\begin{array}{l}\text { Estudo de coorte, } \\
\text { longitudinal, prospetivo e } \\
\text { observacional. A amostra } \\
\text { é constituída por } 34 \\
\text { pessoas sujeitas a } \\
\text { transplante hepático. }\end{array}$ & $\begin{array}{l}97 \% \text { das pessoas apresentam desnutrição e a } \\
\text { mediana do tempo de jejum correspondeu a } \\
13,5 \text { horas. A maioria dos procedimentos } \\
\text { cirúrgicos ocorreram à tarde e a ingestão de } \\
\text { um pequeno-almoço reforçado evitaria } 45 \% \\
\text { de casos de jejum prolongado. }\end{array}$ \\
\hline $\begin{array}{l}\text { Short, V., } \\
\text { Atinkson, C., } \\
\text { Ness, A.R., } \\
\text { Thomas, S., } \\
\text { Burden, S., \& } \\
\text { Sutton, E. / } 2016\end{array}$ & $\begin{array}{l}\text { Patient experiences of } \\
\text { perioperative nutrition } \\
\text { within an enhanced } \\
\text { recovery after surgery } \\
\text { programme for } \\
\text { colorectal surgery: a } \\
\text { qualitative study }\end{array}$ & $\begin{array}{l}\text { Estudo qualitativo e } \\
\text { observacional a envolver } \\
60 \text { clientes submetidos a } \\
\text { cirurgia colorretal. }\end{array}$ & $\begin{array}{l}\text { A ausência de aplicação do protocolo ERAS } \\
\text { levou as pessoas a obterem muita informação } \\
\text { repetida, algumas contraditórias e outras } \\
\text { desnecessárias acerca do jejum pré- } \\
\text { operatório, das bebidas com carboidratos e } \\
\text { da dieta pós-operatória. Isto constituiu uma } \\
\text { barreira ao êxito do processo cirúrgico. A } \\
\text { interação positiva com a equipa, a } \\
\text { acessibilidade a alimentos e à sua escolha e a } \\
\text { motivação para a mudança são facilitadores } \\
\text { para o processo de melhoria. }\end{array}$ \\
\hline $\begin{array}{l}\text { Tosun, B., Yava, } \\
\text { A., \& Açikel, C. / } \\
2015\end{array}$ & $\begin{array}{l}\text { Evaluating the effects } \\
\text { of preoperative fasting } \\
\text { and fluid limitation }\end{array}$ & $\begin{array}{l}\text { Estudo observacional, } \\
\text { transversal, com uma } \\
\text { amostra constituída por } \\
99 \text { pessoas submetidas a } \\
\text { colecistectomia } \\
\text { laparoscópica. }\end{array}$ & $\begin{array}{l}\text { O intervalo de tempo de jejum pré-operatório } \\
\text { encontra-se entre } 3,14 \text { horas e } 14,70 \text { horas. } \\
\text { As pessoas estudadas revelaram possuir } \\
\text { ansiedade moderada em } 58.6 \% \text { dos casos. E } \\
\text { aqueles que tiveram um período de jejum de } \\
12 \text { horas ou mais obtiveram mais fome, sede, } \\
\text { náuseas, dor e níveis de ansiedade e } \\
\text { desconforto significativamente mais } \\
\text { elevados. }\end{array}$ \\
\hline
\end{tabular}

\section{RESULTADOS}

A amostra foi constituída por dez artigos: dois artigos foram desenvolvidos na Turquia, dois nos Estados Unidos da América, dois no Reino Unido e um no Quénia, Chile e Omã. Todos os artigos foram publicados no idioma inglês com exceção de um que se encontrava em língua espanhola. Relativamente ao ano das publicações, observamos maior incidência no ano de 2017 com quatro artigos, seguido de 2016 com três publicações e 2015, 2018 e 2019 identificámos apenas, em cada ano, uma publicação.

No seguimento dos resultados encontrados, com a análise realizada aos artigos selecionados, organizamos os dados em três macrocategorias: orientações sobre o jejum pré-operatório; ganhos inerentes à implementação das orientações sobre o jejum pré- operatório e por fim, fatores dificultadores da implementação das orientações sobre o jejum préoperatório.

\section{Orientações sobre o jejum pré-operatório}

Em oposição aos pressupostos do paradigma do NPO que alegavam contribuir para a minimização do risco de broncoaspiração, surgiram estudos que revelam que o esvaziamento gástrico de alimentos sólidos ocorre por volta de uma hora após a refeição (Maqbali, 2016) tornando-se desnecessário tempos prolongados de jejum. Por outro lado, Çakar, Yilmaz, Çakar, \& Baydur (2017) revelam que a ingestão de líquidos até duas horas antes da intervenção cirúrgica não aumentou o volume gástrico e a acidez do suco gástrico, pelo que não implicou um aumento do risco de aspiração pulmonar. 
Jejum pré-operatório no cliente submetido a cirurgia eletiva: revisão integrativa da literatura

Por outro lado, o recurso a bebidas ricas em carboidratos a anteceder o momento cirúrgico tem constituído uma recomendação a introduzir nos protocolos sobre o jejum pré-operatório contemplando a ingestão destes suplementos até duas horas antes da cirurgia (Noba \& Wakefield, 2019; Tosun, Yara \& Açikel, 2015).

Vários autores defendem que o tempo de esvaziamento destas bebidas é cerca de noventa minutos, não trazendo aumento do risco de broncoaspiração, além de diminuir a resistência à insulina (Noba, \& Wakefield, 2019; Çakar, Yilmaz, Çakar, \& Baydur, 2017).

Neste contexto, os achados encontrados vêm sustentar o proposto pelo protocolo ERAS (2005) que indicava a ingestão de alimentos sólidos integrados numa dieta leve até às seis horas prévias à cirurgia e de líquidos como chã, café, sumos de fruta sem polpa nas duas horas que antecedem a intervenção. Importa ainda referir a possibilidade de se optar pela ingestão oral de $400 \mathrm{ml}$ de água com maltodextrina a $12,5 \%$ até duas horas antes de um procedimento cirúrgico eletivo, mesmo em clientes diabéticos tipo 2 com valores glicémicos controlados.

No que concerne à pessoa com aporte de nutrição entérica, Powers (2017) afirma que o período de suspensão da mesma deve ser limitado ao mínimo possível, não havendo um aumento nos eventos adversos entre os clientes que suspenderam a nutrição entérica no momento anterior à anestesia e os que tinham efetuado suspensão oito horas antes.

Ganhos inerentes à implementação das orientações sobre o jejum pré-operatório
Esta revisão da literatura permitiu perceber que existe unanimidade nos estudos sobre os ganhos inerentes à aplicação das orientações sobre o jejum préoperatório, tanto para a pessoa intervencionada como para a instituição de saúde onde se realiza a cirurgia, pois, cumprindo as guidelines inerentes a esta temática, concorremos para a redução de complicações pós-operatórias e a manutenção do tempo de internamento do doente, dentro daquilo que é o expectável. Por outro lado, no que concerne ao exercício profissional do enfermeiro a existência deste tipo de orientações permitir-lhe-á a conceção e execução de cuidados centrados no cliente, respondendo assim às suas reais necessidades.

Neste contexto, os clientes submetidos a jejum prolongado estão predispostos a alterações no seu bem-estar físico e psicológico. A existência de eventos como o stresse, a desidratação, a hipotensão, as cefaleias, a irritabilidade, a ansiedade, a fome, a sede, a xerostomia, a fadiga, a perda de fluídos durante a cirurgia potenciará o agravamento da desidratação na fase pós-operatória. Por outro lado, o jejum préoperatório prolongado potencia situações de desequilíbrio hidroeletrolítico, contribuindo para a ocorrência de náuseas e vómitos no pós-operatório (Çakar, Yilmaz, Çakar, \& Baydur, 2017).

Constatou-se assim que a diminuição do jejum préoperatório para o intervalo de tempo recomendado produz uma diminuição da fome, sede, cefaleias, desidratação, hipovolémia e náusea em comparação com o paradigma anterior. Concorre também para a diminuição da resistência à insulina, das náuseas e vómitos e do desconforto após a intervenção cirúrgica (Tosun, Yara \& Açikel, 2015). Por outro lado, reduz o 
Jejum pré-operatório no cliente submetido a cirurgia eletiva: revisão integrativa da literatura

risco de desidratação, náuseas e vómitos pósoperatórios, fome, sede e o desconforto físico e psíquico da pessoa (Noba, \& Wakefield, 2019; Çakar, Yilmaz, Çakar, \& Baydur, 2017).

Noba, \& Wakefield (2019) e Çakar, Yilmaz, Çakar, \& Baydur (2017) corroboram os achados anteriores, acrescentam ainda que tem benefícios moderados na redução do tempo de internamento hospitalar e no consumo de terapêuticas farmacológicas em clientes que cumpriram as recomendações atuais com impacto nos custos associados para a instituição de saúde.

\section{Fatores dificultadores da implementação das} orientações sobre o jejum pré-operatório

O processo relativo à implementação das orientações associadas ao jejum pré-operatório têm sido alvo de diversos obstáculos. A informação veiculada ao cliente e como é interpretada tem sido descrita como uma barreira ao correto cumprimento destas orientações, na medida em que a mesma pode não ser transmitida na totalidade pelos profissionais de saúde, ou não é suficientemente clara para permitir ao cliente a sua perceção e aplicação rigorosa na fase de preparação cirúrgica (Short et al., 2016).

A inexistência de protocolos nas instituições de saúde com referência às orientações atuais sobre este tema tem gerado um desalinhamento na forma de atuação dos diversos profissionais de saúde. Por outro lado, de acordo com Munsterman \& Strauss (2018) e Maqbali (2016) foi notória uma incompreensão e renitência na aplicação das orientações mais atuais sobre o jejum pré-operatório por parte de todos os envolvidos. Outras razões são apontadas por Njoroge, Kivuti-Bitok, \& Kimani (2017) como limitadores à implementação destas orientações: a sobrecarga de trabalho dos profissionais, a escassez de tempo para a capacitação do cliente sobre as especificidades deste cuidado e a resistência à mudança de orientações.

\section{DISCUSSÃO}

É conhecido que a pessoa que vai ser submetida a cirurgia, em particular do foro digestivo, se encontra frequentemente desnutrida e desidratada, além de possuir patologias respiratórias, circulatórias e metabólicas variadas que constituem um risco acrescido de má cicatrização, alteração nas anastomoses, infeções pós-operatórias com aumento da morbilidade e mortalidade pós-operatória (Lucchesi, \& Gadelha, 2019). Assim sendo, justifica-se fortemente a necessidade de otimizar a condição da pessoa na fase pré-operatória para reduzir estes riscos. A necessidade de se eliminar barreiras instaladas, no que respeita ao jejum prolongado nos clientes submetidos a cirurgia e praticados sem sustentação científica na prática diária é premente. Vários autores defendem como imperioso a alteração de intervenções de forma a que o consumo de alimentos sólidos seja realizado até seis horas antes do procedimento, e de líquidos até duas horas antes, introduzindo-se ainda o fornecimento de suplementos carboidratados (Diógenes, Costa, \& Rivanor, 2019; Campos, Barros-Neto, Guedes, \& Moura, 2018), contemplando o ajuste do seu início, mediante a hora da cirurgia (Diógenes, Costa, \& Rivanor, 2019) e de acordo com patologias de cada cliente. Desta forma, os cuidados direcionam-se para as necessidades individuais de cada cliente, contribuindo para minimizar possíveis sentimentos de stresse e angústia, vivenciados pelo cliente, associados ao evento pelo 
Jejum pré-operatório no cliente submetido a cirurgia eletiva: revisão integrativa da literatura

qual irá passar. Na verdade, estas mudanças nos contextos da prática permitem a obtenção de ganhos para a pessoa intervencionada ao nível físico e psicológico, de que é exemplo a diminuição das complicações pós-operatórias e a recuperação mais precoce, assim como para a instituição de saúde, nomeadamente a redução do tempo de internamento. O empoderamento dos profissionais de saúde sobre estas temáticas torna-se uma ferramenta indispensável para a adoção correta das orientações emanadas (Santo et al., 2020), permitindo também o envolvimento dos clientes, neste processo, e levando ao sucesso na implementação de tais orientações. Efetivamente, os enfermeiros têm um papel diferenciador em todo este processo, dada a sua proximidade com o cliente (Chaves, \& Campos, 2019), permitindo um maior nível de eficiência na prática de cuidados (Silva et al., 2019). Cabe ao enfermeiro desmistificar a ideia presente no contexto social, e fortemente enraizada, de que o jejum deverá ser a partir das zero horas do dia da cirurgia (Diógenes, Costa, \& Rivanor, 2019), através da consciencialização do cliente sobre este procedimento pré-operatório.

Por outro lado, o facto de terem um papel de destaque no processo de cuidados dos clientes, permite-lhe a criação de normas que aliem a melhor evidência com as especificidades logísticas e organizacionais de cada serviço/instituição (Santo, et al., 2020).

Assumimos que a baixa flexibilidade e motivação dos profissionais para a mudança de práticas é um fator contributivo para, ainda hoje, em se manter desatualizadas as orientações, além do envolvimento insuficiente dos serviços no desenvolvimento da prática baseada na evidência.
Corroborando Miguez, Silva, \& Oliveira (2019), apelamos à existência de normas sobre este tema para direcionar uniformemente a prática dos profissionais de saúde, dado que em grande parte dos serviços hospitalares encontram-se desajustados do conhecimento atual, perpetuando o conceito NPO após as zero horas do dia da cirurgia.

No nosso estudo, uma das limitações prendeu-se com a ausência de investigações da realidade portuguesa, não a permitindo conhecer, no que concerne às recomendações associadas ao jejum pré-operatório. A atuação do enfermeiro ficará dificultada na eliminação de lacunas da prática diária que geram prejuízos ao cliente e instituição. Sugere-se a realização de estudos no contexto nacional que relacionem a aplicação das orientações descritas nesta revisão com a redução de complicações cirúrgicas e de que modo podem ser traduzidos em ganhos associados aos cuidados de saúde.

\section{CONCLUSÃO}

O jejum pré-operatório adequado à condição clínica do cliente deverá ser uma estratégia positiva capaz de minimizar o impacto das comorbilidades. Contribui também para a diminuição de complicações operatórias, do período de hospitalização, a redução dos custos em saúde e o aumento da sua satisfação em relação aos cuidados de saúde. As orientações para a implementação do jejum pré-operatório preconizam uma redução do período de abstinência para alimentos sólidos e líquidos, respetivamente, até seis horas e duas horas antes da cirurgia, além do recurso a bebidas carboidratadas. 
No desempenho profissional do enfermeiro deverá ser tido em conta as contribuições deste estudo, na medida em que constitui uma base na sistematização dos cuidados de enfermagem direcionados ao cliente, enaltecendo o papel dos enfermeiros, através da melhoria contínua dos cuidados de enfermagem, com ganhos para o cliente e para a instituição.

\section{REFERÊNCIAS BIBLIOGRÁFICAS}

Aguilar-Nascimento, J., Dias, A. L. A., Dock-Nascimento, D. B., Correia, M. I. T. D., Campos, A. C. L., Portari-Filho, P. E., \& Oliveira, S. S. (2014). Atual preoperative fasting time in Brazilian hospitals: The BIGFAST multicentre study. Therapeutics and clinical risk Management, 10, 107-112. DOI: 10.2147/TCRM.556255

Botelho, L., Cunha, C., \& Macedo, M. (2011). O método da revisão integrativa nos estudos organizacionais. Gestão e sociedade, 5(11), 121-136. DOI: 10.21171/ges.v5i11.1220

Çakar, E., Yilmaz, E., Çakar, E., \& Baydur, H. (2017). The effect of preoperative oral carbohydrate solution intake on patient Comfort: A randomized controlled study. Journal of Perianesthesia Nursing: official journal of the American Society of PeriAnesthesia Nurses, 32(6), 589-599. DOI: 10.1016/j.jopan.2016.03.008

Campos, S. Barros-Neto, J. A., Guedes, G., \& Moura, F. A. (2018). Pre-operative fasting: Why abbreviate? Arquivos brasileiros de cirurgia digestiva: $A B C D=$ Brazilian archives of digestive surgery, 31(2), e1377. DOI: 10.1590/0102-672020180001e1377

Chaves, L. M., \& Campos, J. S. P. (2019). Abreviação do jejum e suporte nutricional pré-operatório em cirurgias eletivas: Uma revisão sistemática. Revista Eletrônica Acervo Saúde, (35), e2210. https://doi.org/10.25248/reas.e2210.2019

Diógenes, D.H., Costa, C.S.D., \& Rivanor, R.L.D.C. (2019). Fasting Time in the Preoperative of Elective Surgeries at a Referral Hospital in Trauma in the Municipality of Fortaleza - CE.Revista Brasileira de Ciências da Saúde, 23(2), 191-196. DOI: 10.22478/ufpb.2317-6032.2019v23n2.32118

Francisco, S.C., Batista, S. T., \& Pena, G.G. (2015). Jejum em pacientes cirúrgicos eletivos: comparação entre o tempo prescrito, praticado e o indicado em protocolos de cuidados perioperatórios. Brazilian archives of digestive surgery, 28(4), 250-254. DOI: 10.1590/s01026720201500040008.

Lucchesi, F.D.A., \& Gadelha, P.C.F.P. (2019). Nutritional status and evaluation of the perioperative fasting time among patients submitted to elective and emergency surgeries at a reference hospital. Revista do Colégio Brasileiro de Cirurgiões, 46(4), e20192222. DOI: 10.1590/0100-6991e-20192222

Maqbali, M. A. A. (2016). Preoperative fasting for eletive surgery in a regional hospital in Oman. British Journal of Nursing, 25(14), 798-802. DOI: 10.12968/bjon.2016.25.14.798

Miguez, B.B., Silva, A.H., \& Oliveira, A.P. (2019). The importance of reducing pre-operative fast time: a literary review. Cadernos da Medicina-UNIFESO, 2(2), 174-182. Retirado de: http://www.revista.unifeso.edu.br/index.php/cadern osdemedicinaunifeso/article/view/1389/604

Munsterman, C. \& Strauss, P. (2018). Early rehydration in surgical patients with prolonged fasting decreases postoperative nausea and vomiting. Journal of Perianesthesia Nursing: official journal of the American Society of PeriAnesthesia Nurses, 33(5), 629-631. DOI: 10.1016/j.jopan.2017.06.124

Njoroge, G., Kivuti-Bitok, L., \& Kimani, S. (2017). Preoperative fasting among adult patients for elective surgery in a Kenyan Referral Hospital. International Scholarly Research Notices, 2017, 1-8. DOI: 10.1155/2017/2159606

Noba, L., \& Wakefield, A. (2019). Are carbohydrate drinks more effective than preoperative fasting: A systematic review of randomised controlled trials. Journal of clinical nursing, 28(17-18), 3096-3116. DOI: 10.1111/jocn.14919

Pinto, A.S., Grigoletti, S.S., \& Marcadenti, A. (2015). Abreviação do jejum entre pacientes submetidos à cirurgia oncológica: revisão sistemática. Brazilian archives of digestive surgery, 28(1), 70-73. DOI:10.1590/50102-67202015000100018.

Powers, J. (2017). Guidelines for preoperative fasting for hospitalized patients. Critical care nurse, 37(5), 9092. DOI: $10.4037 / \operatorname{ccn} 2017778$

Sánchez, A., \& Papapietro, K. (2017). Nutrición perioperatoria en protocolos quirúrgicos para una mejor recuperación postoperatoria (Protocolo ERAS). 
Revista Médica do Chile, 145(11), 1447-1453. DOI: 10.4067/s0034-98872017001101447

Santo, I.M.B.E., Matos, J.C., Silva, C.J., Almeida, R.P., Santos, J.L.P., Silva, S.M., ... \& Barbosa, S.S. (2020). Sistematização da Assistência de Enfermagem Perioperatória (SAEP): Reflexos da Aplicabilidade no Processo de Cuidar. Revista Eletrônica Acervo Saúde, (43), e2945. DOI: 10.25248/reas.e2945.2020

Short, V., Atkinson, C., Ness, A.R., Thomas, S., Burden, S., \& Sutton, E. (2016). Patient experiences of perioperative nutrition within an Enhanced Recovery After Surgery programme for colorectal surgery: a qualitative study. Colorectal Disease, 18(2), 74-80. DOI: 10.1111/codi.13245

Silva, C., Soares, L., Ferreira, M.A., Jesus, S., Príncipe, F. \& Mota, L. (2019). Representação social do enfermeiro sobre a visita no pós-operatório em clientes cirúrgicos. Revista De Investigação \& Inovação Em Saúde, 2(2), 4757. DOI: 10.37914/riis.v2i2.58

Sousa, L.M.M., Firmino, C.F.F., Marques-Vieira, C.M. A., Severino, S.S.P., \& Pestana, H.C.F.C. (2018). Reviews of the scientific literature: types, methods and applications in nursing. Revista Portuguesa de Enfermagem de Reabilitação, 1(1), 45-54. Retirado de: https://www.aper.pt/ficheiros/revista/rperv1n1.pdf
Souza, M.T.D., Silva, M.D.D., \& Carvalho, R.D. (2010). Revisão integrativa: o que é e como fazer. Einstein (São Paulo), 8(1), 102-106. DOI: 10.1590/s167945082010rw1134

Tosun, B., Yava, A., \& Açikel, C. (2015). Evaluating the effects of preoperative fasting and fluid limitation. International journal of nursing practice, 21(2), 156165. DOI: 10.1111/ijn.12239

Vidot, H., Teevan, K., Carey, S., Simon, S., \& Shackel, N. (2016). A prospective audit of preprocedural fasting practices on a transplant ward: when fasting becomes starving. Journal of clinical nursing, 25(5-6), 829-835. DOI: 10.1111/jocn.13116

Vilelas, J. (2017). Investigação: o processo de construção do conhecimento. (2ªed). Lisboa: Edições Sílabo, 2017.

Yildiz, H., Gunal, S.E., Yilmaz, G., \& Yacel, S. (2013). Oral carbohydrate supplementation reduces preoperative discomfort in laparoscopic cholecystectomy. Journal of investigative surgery: the oficial journal of the Academy of Surgical Research, 26(2), 89-95. DOI: 10.3109/08941939.2012.699998 